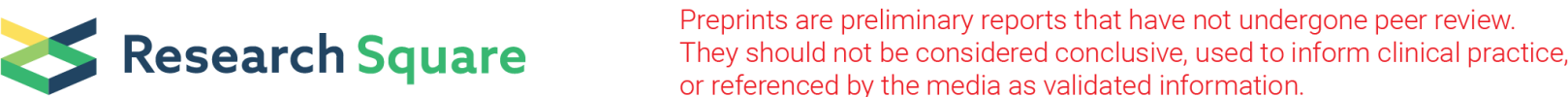

\section{Changes in Retinal and Choroidal Vascularity in Eyes with Acute Central Serous Chorioretinopathy Using Optical Coherence Tomography Angiography}

Jingli Guo

EYE andENT OF Fudan University

\section{Xinyi Ding}

EYE and ENT hospital of Fudan University

Yongjin Zhang

EYE and ENT hospital of Fudan University

Gezhi Xu

EYE and ENT hospital of Fudan University

Haixiang Wu ( $\nabla$ whx577@163.com )

\section{Research article}

Keywords: Acute central serous chorioretinopathy; ellipsoid zone disruption; flat pigment epithelial detachment; microvascular density; optical coherence tomography angiography

Posted Date: March 19th, 2019

DOl: https://doi.org/10.21203/rs.2.459/v1

License: (c) (i) This work is licensed under a Creative Commons Attribution 4.0 International License. Read Full License 


\section{Abstract}

Background To compare the vascular changes of superficial capillary layer (SCP) and deep capillary layer (DCP) in retina and choriocapillary layer in eyes with acute central serous chorioretinopathy (CSCR) by optical coherence tomography angiography (OCTA) between at baseline and 3 months. Methods Prospective case series; Twelve patients (12 eyes) with acute CSCR at the baseline and 3 months were included. All patients underwent comprehensive ophthalmic examinations. Subfoveal choroidal thickness (SFCT) and central macular thickness (CMT) were evaluated by swept domain optical coherence tomography (SD-OCT). The foveal avascular zone (FAZ), the microvascular morphology and density of SCP and DCP, and the choroicapillary morphology were assessed by OCTA and Image $\mathrm{J}$ software. Results The mean CMT $(p=0.018)$, the mean SFCT $(p=0.013)$, the mean microvascular density of DCP $(p<0.001)$ and choroicapillary layer $(p<0.001)$ at baseline were different from the ones at 3 months. The density of DCP was increased and the areas of choriocapillaris flow signal void was shrinked at 3months in the process of self-resolve. Two eyes exhibited flat pigment epithelial detachment by OCT and OCTA demonstrated the formation of vascular in choroicapillary layer at 3 months. The en-face OCT can illstrate the area of ellipsoid zone (EZ) disruption. Conclusions OCTA enables the visualization of microvascular features of the DCP and choroidcapillary in eyes with acute CSCR in the process of selfresovle to help elucidate the pathophysiology. Flat pigment epithelial detachment presented by OCT in acute CSCR eyes can be revealed vascular structure in choroicapillary layer by OCTA. OCTA imaging seems to be a useful tool in the identification of acute CSCR.

\section{Background}

Acute central serous chorioretinopathy (CSCR) is a choroiretinopathy disorder characterized by limited serous detachments of the neurosensory retina and usually combined with focal pigment epithelial detachments (PED) on account of choroidal hyperpermeability, dilated choroidal vessels and retinal pigment epithelial (RPE) barrier breakdown. ${ }^{1}$ Color photograph presents oval lesions in the macular. ${ }^{2}$ Swept domain optical coherence tomography (SD-OCT) is the primary imaging modality for the diagnosis and follow-up of CSCR, ${ }^{3}$ Enhanced-depth imaging (EDI) have supplied full-depth visualization of the choroid, enhancing the morphological analysis of the vessels in choroidal capillary and Haller's layer and choroidal thickness. ${ }^{3}$ Fundus autofluorescence (FAF) could reflect RPE function state and allow a noninvasive detection of a series of alterations at different phases and types of CSCR. ${ }^{4,5}$ Fluorescein fundus angiography (FFA) indicates the origin of leakage of acute CSCR. ${ }^{6}$ In spite of not compulsiveness for the diagnosis of CSCR, but FFA is helpful to confirm the diagnosis and provides a guide for possible photodynamic therapy of eccentric leaks. ${ }^{7}$ Indocyanine green angiography (ICGA) has become the gold standard of the visualization of the choroidal vasculature and CNV complicating CSCR. ${ }^{8}$ However, FFA and ICG have not only both invasive examations and a spetrum of contraindication, but also not suitable for follow-up observation of microvascular changes of retina and choroicapillary. 
Optical coherence tomography angiography (OCTA) is a new-noninvasive imaging modality that allows the visualization of blood flow of retina and choroicapillary, appling to evaluate retinal vascular disease. ${ }^{9-}$ ${ }^{11}$ Signal strength detected by OCTA is positively correlated with blood perfusion. ${ }^{12}$ Recently, several studies used OCTA to assess the presence of CNV in CSCR with variable and noncomparable results. ${ }^{13,14}$ However, less research focus on the microvascular changes of retina and choroicapillary in eyes with acute CSCR. ${ }^{15}$ Because serous retinal detachments resolve spontaneously within three months in most acute CSCR episodes, ${ }^{1}$ the ideal timing for interventions still remains to be determined. Moreover, it can further supply more morphological informations for exploring the mechanisms of acute CSCR.

On account of the previous research about the structure changes of retina and choroid by OCT, the aim of this study is to further explore the microvascular changes of retina and choriocapillary in eyes with aute CSCR by OCTA during the process of self-resolve.

\section{Methods}

This was a prospective case series studies. 12 consecutive patients with acute CSCR were enrolled from September 2016 to December 2017 at the outpatient clinic of the Eye \& ENT Hospital of Fudan University, Shanghai, China. Informed consent was obtained from all patients. All procedures were performed according to the principles of the Declaration of Helsinki.

Comprehensive ocular examinations was performed for all patients in both eyes, involving slit-lamp biomicroscopic ophthalmoscopic, best-corrected visual acuity (BCVA), dilated fundoscopy, fundus photography (Topcon TRC50LX; Topcon, Tokyo, Japan), intraocular pressure (IOP), SD-OCT (Heidelberg Engineering, Heidelberg, Germany) and OCTA (Optovue RTVue XR 100 Avanti, Fremont, CA, USA). All patients in this study was included without any cular treatment previously.

Acute CSCR was defined by the presence of serous retinal detachment within 3 months. The clinical criteria used for diagnosing acute CSCR were confirmed by OCT and OCTA, including the following: (1) the onset of visual symptoms (vision impairment, metamorphopsia, micropsia, dyschromatopsia or central scotoma) within 3 months, (2) the contralateral eyes without any anterior and posterior disorders, (3) SD-OCT detection of serous retinal detachment involving the fovea, increased choroidal thickness and dilated choroidal vessels, (4) OCTA detection of focal high-intensity lesion indicating abnormal choroidal vessels and flow void lesion at the choriocapillaris layer and (5) The signal strength index of all images by OCTA was greater than 60 without disordered layers.(6) the symptom improved and subretinal fluid resolved at 3 months. Exclusion criteria were patients with spherical error superior to $2 \mathrm{D}$, amblyopia or anisometropia, cataracts, combined with history of other ophthalmic diseases and ocular trauma, the history of laser and surgery and drug therapy. The follow-up scheme for this observational study included repeated monthly clinical evaluation for 3 months.

SD-OCT was performed as previous mode, as well as the evaluation of CMT. ${ }^{16}$ The subfoveal choroidal thickness (SFCT) was measured using enhanced-depth imaging scans as the axial distance from the RPE 
to the sclera interface. All images were obtained by two independent well-trained operators.

Observers assessed the OCTA data using the best-quality $3 \times 3-\mathrm{mm}$ scan and controlled the corrected segmentation for the 12 patients before reporting the data. Vascular retinal layers were divided into the SCP, DCP, outer retina and choroicapillary layers (CC) by OCTA. The FAZ and density of the macula were assessed using flow density map software Angio-Analytics (Optovue RTVue XR 100 Avanti). The density of choroicapillary was assessed by Image $\mathrm{J}$ software. Whole-image data were used to measure the microvascular densities in the SCP and DCP layers.

\section{Statistical Analysis}

Visual acuity were incorporation into logarithm of the minimum angle of resolution (logMAR) for statistical analysis. Quantitative data (mean BCVA, CMT, vascular density of SCP, DCP and choroicapillary layer, FAZ areas of the SCP) were compared between at baseline and 3 months by the Mann-Whitney test using IBM SPSS Statistics v19 (SPSS Inc., Chicago, IL, USA). Significance was defined as P $<0.05$.

\section{Results}

A total of 12 eyes in 12 patients ( 8 men and 4 women) were included in the study. The demographic characteristics of the patients are listed in Table 1. The mean \pm SD age was $45.50 \pm 9.69$ years (range, 2563 years). The BCVA values (mean \pm SD) in the eyes with acute CSCR at baseline (logMAR) were $(0.27 \pm 0.23)$ (range, $20 / 25$ to $20 / 100)$, and at 3 months $(0.08 \pm 0.15)$ (range, $20 / 20$ to $20 / 50$ ), respectively $(p=0.03)$. The CMT values (mean \pm SD) in the eyes with acute CSCR at baseline and 3 months were $(420.33 \pm 123.43) \mu \mathrm{m},(296.42 \pm 114.01) \mu \mathrm{m}(p=0.018)$, respectively (Table 1). The SFCT values (mean \pm SD) in the eyes with acute CSCR at baseline and 3 months were $(376.42 \pm 72.66) \mu \mathrm{m},(280.08 \pm 100.61) \mu \mathrm{m}$ $(p=0.013)$.

The FAZ areas of the SCP were $(0.33 \pm 0.11) \mathrm{mm}^{2}$ at baseline and $(0.32 \pm 0.92) \mathrm{mm}^{2}$ at 3 months $(p=0.915)$ in acute CSCR eyes using OCTA software. The mean vessel density value of SCP, DCP and CC were evaluated by OCTA and Image $J$ software respectively (shown in Table 2). The morphology of SCP, DCP and CC was presented by OCTA , comparing the microvascular changes, and the extent and range of angiectasis at baseline and 3 months. (shown in Figure 1) The range of subretinal fluid and the areas of IS/OS disruption were evaluated by en-face imaging. (shown in Figure 2). Two eyes exhibited flat pigment epithelial detachment by OCT and OCTA demonstrated the formation of vascular in choroicapillary layer at 3 months. (shown in Figure 3) The microvascular density of DCP corresponding to the high signal in the CC layer was recovered at 3 months. (shown in Figure 4)

\section{Discussion}

In acute CSCR, OCTA could be a potentially useful imaging modality for providing a real-time direct visualization of the blood flow in the SCP, DCP and CC in the process of self-resolve, contributing to provide theoretical basis for the mechanism of acute CSCR. In a previous study, based on dye 
angiography combined with SD-OCT, we were only able to identify subretinal fluid and leakage of RPE. However, in the present study, OCTA can enhance to detect the microvascular morphology and density of SCP, DCP and CC at baseline and 3 months. Although the key mechanisms of acute CSCR are choroidal vasodilation and leakage through the RPE, the choroidal circulation is not only for the choroid, but also for the RPE and as far as the lateral part of the retinal inner layer (ie, the deep capillary region). ${ }^{17,18}$ Birol et al. confirmed in an animal model that the deep capillary bed is conducive to the oxygen requirements of the photoreceptor layer. ${ }^{18}$ In their study, the primary oxygen supply to photoreceptor layers was derived from the choroidal circulation, but $10 \%-15 \%$ was derived from the retinal circulation. ${ }^{19}$ As oxygenation of the fovea is somewhat different from that of the perifoveal retina, it would be useful to discuss this and any other potentially relevant correlations regarding the foveal and perifoveal areas. ${ }^{18}$ From these results, the microvascular changes of DCP and choroicapillary could contribute to prognosis determination important target. In our study, the density of DCP and choroicapillary increased at 3 months, which proved the recovery of acute CSCR.

Chan et $\mathrm{al}^{20}$ retrospectively reported 12 eyes with CSCR presented foci high signal in choroicapillary layer by OCTA, demonstrating the dilated choroicapillary. It is concluded that OCTA can be used as an auxiliary tool to diagnose CSCR. In contrast, we observed changes in the vasoconstriction of the choroicapillary dilatation during the recovery of acute CSCR patients. Although the subretinal fluid was completely absorbed detected by OCT, choroicapillary with high signal can still be remained by OCTA. This phenomenon, related stimuli, such as smoking, alcoholism, staying up late and so on, explains why many patients with acute CSCR are prone to recurrence. Therefore, with the advantage of OCTA in the follow-up process, it is possible to extend the follow-up treatment time after the complete recovery of choroidal capillaries, which indicates that OCTA provides an effective reference for clinical follow-up treatment. Teussink et $\mathrm{al}^{21}$ compared 18 patients with chronic CSCR with 6 nomal controls who underwent FFA, ICGA and OCTA, illstrating the same presentation of choroicapillary by OCTA as the ones by ICGA. Refered to the aboved research, the main diagnosis were: less than 3 months of onset, subretinal fluid accumulation by OCT, and squamous high signal in choroicapillary layer presented by OCTA in our study.

The changes of microvascular density in different layers are diverse not only among various retinal diseases, but also the progress or efficacy of disease. ${ }^{22-25}$ For example, the extend of photoreceptor damage is related to the blood flow density of DCP in diabetic retinopathy. ${ }^{24,25}$ Moreover, another publication showed reduced microvascular density of SCP and the increased density of DCP in adultonset foveomacular vitelliform dystrophy. ${ }^{23}$ At present, rare study is focus on the microvascular density in SCP, DCP and CC in acute CSCR episodes without treatment. Nelis et $\mathrm{al}^{27}$ compared 16 eyes with acute CSCR with contralateral eyes and normal eye, resulting in the much more density of SCP and much smaller of FAZ in eyes with acute CSCR than the contralateral and normal eyes. However, the higher density of DCP in 12 eyes with acute CSCR at 3 months compared with eyes at initial visit, but no significant differene in density and FAZ of SCP between at baseline and at 3months. At the same time, in spite of completely absorption of subretinal fluid, the photoreceptor layer need longer time to recovery. We could identify the disruption of IS/OS by en -face imaging, which is related with the visual acuity. 
Elevation of RPE by OCT was found in $19-68 \%$ in acute CSCR patients, ${ }^{3}$ but few studies focus on the vascular changes of CC caused by flat PED in eyes with acute CSCR. In our study, two eyes with acute CSCR exhibited flat pigment epithelial detachment by OCT and OCTA demonstrated the formation of vascular in choroicapillary layer at 3 months.

Several improvements are needed: the larger sample size; OCTA underwent contemporaneously with FFA/ICGA in order to determine more accurately the relative sensitivities of each of these modalities;Longitudinal studies are needed to describe the microvascular changes of retina and choroid.

\section{Conclusions}

OCTA can be used as an important aid in the diagnosis and follow-up of acute CSCR. Through observation during follow-up period, the more increased microvascular density of DCP and CC in patients with acute CSCR was showed after subretinal fluid improved or resolved. The extend of disruption at the IS/OS level can be initially determined by en-face OCT, which is helpful for providing a good basis for visual observation during follow-up.

\section{Abbreviations}

BCVA: best-corrected visual acuity; CC: choroicapillary layers; CSCR: central serous chorioretinopathy; DCP: deep capillary layer; EDI: enhanced-depth imaging; FAF: fundus autofluorescence; FFA: fluorescein fundus angiography; ICGA: indocyanine green angiography; OCTA: optical coherence tomography angiography; PED: pigment epithelial detachments; RPE:retinal pigment epithelial; SCP: superficial capillary layer; SD-OCT: swept domain optical coherence tomography; SFCT: subfoveal choroidal thickness;

\section{Declarations}

Ethics approval and consent to participate This study was approved by the Institutional Review Board of the Eye and ENT Hospital of Fudan University, Shanghai, China. All procedures were approved by the principles of the Declaration of Helsinki.Written informed consent was obtained from all participants or their guardians

Availability of data and materials Transcripts from this study are available for sharing upon request; however, all identifying and confidential information of the participants will be removed.

\section{Consent for publication Not Applicable}

Competing interests All authors certify that they have no affiliations with or involvement in any organization or entity with any financial interest (such as honoraria; educational grants; participation in 
speakers' bureaus; membership, employment, consultancies, stock ownership, or other equity interest; and expert testimony or patent-licensing arrangements), or non-financial interest (such as personal or professional relationships, affiliations, knowledge or beliefs) in the subject matter or materials discussed in this manuscript.

Funding This work was supported by funding from the National Natural Science Foundation for Young Scholars of China (81300781). The funder had no role in study design, data collection and analysis, decision to publish, or preparation of the manuscript.

Author contributions JLG: data analysis and manuscript preparation; XYD: analysis and interpretation of data; HXW: concept and design , data acquisition, critical revision for important intellectual content; GZX: concept and design, critical revision for important intellectual content; YJZ: concept and design.

Acknowledgements We would like to thank all of the participants and staff for their valuable contributions to this research

\section{References}

1. Daruich A, Matet A, Marchionno L, De Azevedo JD, Ambresin A, Mantel I, Behar-Cohen F囚2017\ACUTE CENTRAL SEROUS CHORIORETINOPATHY: Factors Influencing Episode Duration. Retina 37:19051915

2. Plateroti AM, Witmer MT, Kiss S, D'Amico DJ (2014) Characteristics of intraretinal deposits in acute central serous chorioretinopathy. Clin Ophthalmol 8:673-676

3. Plateroti AM, Witmer MT, Kiss S, D'Amico DJ (2015) Central serous chorioretinopathy: Recent findings and new physiopathology hypothesis. Prog Retin Eye Res: 48:82-118

4. Spaide R (2008) Autofluorescence from the outer retina and subretinal space: hypothesis and review. Retina 28(1):5-35

5. Choi KE, Yun C, Kim YH, Kim SW, Oh J, Huh K (2017) THE EFFECT OF PHOTOPIGMENT BLEACHING ON FUNDUS AUTOFLUORESCENCE IN ACUTE CENTRALSEROUS CHORIORETINOPATHY. Retina 37:568-577

6. Eandi CM, Ober M, Iranmanesh R, Peiretti E, Yannuzzi LA (2005) Acute central serous chorioretinopathy and fundus autofluorescence. Retina 25:989-993

7. Yannuzzi LA, Freund KB, Goldbaum M, Scassellati-Sforzolini B, Guyer DR, Spaide RF, Maberley D, Wong DW, Slakter JS, Sorenson JA, Fisher YL, Orlock DA (2000) Polypoidal choroidal vasculopathy masquerading as central serous chorioretinopathy. Ophthalmology 107:767-7

8. Piccolino FC, Borgia L (1994) Central serous chorioretinopathy and indocyanine green angiography. Retina 14:231-242

9. Mao L, Weng SS, Gong YY, Yu SQ (2017) Optical coherence tomography angiography of macular telangiectasia type 1: Comparison with mild diabetic macular edema. Lasers Surg Med 49:225-232 
10. Mo J, Duan A, Chan S, Wang X, Wei W (2017) Vascular flow density in pathological myopia: an optical coherence tomography angiography study. Bmj Open 7(2):e13571

11. Chihara E, Dimitrova G, Amano H, Chihara T (2017) Discriminatory Power of Superficial Vessel Density and Prelaminar Vascular Flow Index in Eyes With Glaucoma and Ocular Hypertension and Normal Eyes. Invest Ophthalmol Vis Sci 58:690-697

12. Koustenis AJ, Harris A, Gross J, Januleviciene I, Shah A, Siesky B (2017) Optical coherence tomography angiography: an overview of the technology and an assessment of applications for clinical research. Br J Ophthalmol 101:16-20

13. Bonini Filho MA, de Carlo TE, Ferrara D, Adhi M, Baumal CR, Witkin AJ, Reichel E, Duker JS, Waheed NK (2015) Association of Choroidal Neovascularization and Central Serous Chorioretinopathy With Optical Coherence Tomography Angiography. JAMA Ophthalmol 133:899-906.

14. McClintic SM, Jia Y, Huang D, Bailey ST (2015) Optical coherence tomographic angiography of choroidal neovascularization associated with central serous chorioretinopathy. JAMA Ophthalmol 133:1212-1214

15. Qu Y, Gong D, Yu W, Dong F (2017) Characteristics of the Choriocapillaris Layer in Optical Coherence Tomography Angiography of Acute Central Serous Chorioretinopathy. Ophthalmic Surg Lasers Imaging Retina 48:1000-1005

16. Guo J, Tang W, Ye X, et al. Predictive multi-imaging biomarkers relevant for visual acuity in idiopathic macular telangiectasis type 1. Bmc Ophthalmol 2018;18(1):69.

17. Staurenghi G, Sadda S, Chakravarthy U, Spaide RF (2014) Proposed lexicon for anatomic landmarks in normal posterior segment spectral domain optical coherence tomography. The IN·OCT consensus. Ophthalmology 121:1572-1578

18. Wakazono T, Ooto S, Hangai M, Yoshimura N (2013) Photoreceptor outer segment abnormalities and retinal sensitivity in acute zonal occult outer retinopathy. Retina 33(3):642-648

19. Birol G, Wang S, Budzynski E, Wangsa-Wirawan ND, Linsenmeier RA (2007) Oxygen distribution and consumption in the macaque retina. Am J Physiol Heart Circ Physiol 293:H1696-704

20. Chan SY, Wang Q, Wei WB, Jonas JB $₫ 2016 \rrbracket$ OPTICAL COHERENCE TOMOGRAPHIC ANGIOGRAPHY IN CENTRAL SEROUS CHORIORETINOPATHY. Retina 36:2051-2058.

21. Teussink MM, Breukink MB, van Grinsven MJ, Hoyng CB, Klevering BJ, Boon CJ, de Jong EK, Theelen T (2015) OCT Angiography Compared to Fluorescein and Indocyanine Green Angiography in Chronic Central Serous Chorioretinopathy. Invest Ophthalmol Vis Sci 56(9):5229-37

22. Mastropasqua R, Toto L, Borrelli E, Di Antonio L, Mattei PA, Senatore A, Di Nicola M, Mariotti C $₫ 2017 \rrbracket$ Optical Coherence Tomography Angiography Findings in Stargardt Disease. Plos One 12:e170343

23. Battaglia Parodi M, Rabiolo A, Cicinelli MV, lacono P, Romano F, Bandello F (2018) QUANTITATIVE ANALYSIS OF OPTICAL COHERENCE TOMOGRAPHY ANGIOGRAPHY IN ADULT-

ONSETFOVEOMACULAR VITELLIFORM DYSTROPHY. Retina 38:237-244

24. Ghasemi FK, lafe NA, Hubschman JP, Tsui I, Sadda SR, Sarraf D (2017) Optical Coherence Tomography Angiography Analysis of the Foveal Avascular Zone and Macular Vessel Density After 
Anti-VEGF Therapy in Eyes With Diabetic Macular Edema and Retinal Vein Occlusion. Invest Ophthalmol Vis Sci 58:30-34

25. Nesper PL, Scarinci F, Fawzi AA (2017) Adaptive Optics Reveals Photoreceptor Abnormalities in Diabetic Macular Ischemia. Plos One 12:e169926

26. Dimitrova G, Chihara E, Takahashi H, Amano H, Okazaki K (2017) Quantitative Retinal Optical Coherence Tomography Angiography in Patients With Diabetes Without Diabetic Retinopathy. Invest Ophthalmol Vis Sci 58:190-196

27. Nesper PL, Scarinci F, Fawzi AA (2017) Adaptive Optics Reveals Photoreceptor Abnormalities in Diabetic Macular Ischemia. Plos One 12:e169926

\section{Tables}

Table 1 Demographics and Clinical Data of Patients with acute CSC at baseline

\begin{tabular}{ccccccc}
\hline Case & Sex & BCVA & Eye & Age, & Time onset, & FIPED \\
& & & & & \\
Ranges & days & \\
1 & M & $20 / 25$ & OD & $41-50$ & 7 & $(-)$ \\
2 & M & $20 / 80$ & OD & $51-60$ & 36 & $(-)$ \\
3 & M & $20 / 25$ & OS & $41-50$ & 14 & $(-)$ \\
4 & F & $20 / 25$ & OD & $31-40$ & 6 & $(-)$ \\
5 & F & $20 / 32$ & OS & $61-60$ & 16 & $(-)$ \\
6 & M & $20 / 25$ & OD & $41-50$ & 10 & $(-)$ \\
7 & F & $20 / 125$ & OD & $51-60$ & 30 & $(-)$ \\
8 & F & $20 / 32$ & OS & $51-60$ & 14 & $(-)$ \\
9 & M & $20 / 25$ & OD & $31-40$ & 5 & $(+)$ \\
10 & M & $20 / 32$ & OS & $41-50$ & 30 & $(+)$ \\
11 & M & $20 / 40$ & OS & $21-30$ & 15 & $(-)$ \\
12 & M & $20 / 50$ & OD & $31-40$ & 30 & $(-)$ \\
\hline
\end{tabular}

BCVA: best-corrected visual acuity; FIPED: flat irregular pigment epithelial detachment;

Time:Time from symptoms onset to first visit

Table 2 OCT and OCTA-derived characteristics of patients with acute CSC at baseline and 3 months

\begin{tabular}{ccccc}
\hline & & At baseline & At 3 months & $p$ value \\
CMT & $\mu \mathrm{m}$ & $420.33 \pm 123.43$ & $296.42 \pm 114.01$ & 0.018 \\
SFCT & $\mu \mathrm{m}$ & $376.42 \pm 72.66$ & $280.08 \pm 100.61$ & 0.013 \\
SCP & FAZ $\left(\mathrm{mm}^{2}\right)$ & $0.33 \pm 0.11$ & $0.32 \pm 0.92$ & 0.915 \\
& Density(\%) & $49.15 \pm 2.54$ & $49.87 \pm 2.01$ & 0.450 \\
DCP & Density(\%) & $53.31 \pm 2.58$ & $56.68 \pm 1.47$ & 0.001 \\
SCP' & Density(\%) & $0.37 \pm 0.09$ & $0.38 \pm 0.04$ & 0.560 \\
DCP' $^{\prime}$ & Density(\%) & $0.38 \pm 0.03$ & $0.43 \pm 0.01$ & $\square 0.001$ \\
CC' $^{\prime}$ & Density(\%) & $0.33 \pm 0.05$ & $0.47 \pm 0.04$ & $\square 0.001$ \\
\hline
\end{tabular}

CC:choriocapillary layer; CMT: central macular thickness; CSC:central serous chorioretinopathy; DCP: deep capillary layer; OCT: optical coherence tomography; OCTA: optical coherence tomography angiography; SCP: 
superficial capillary layer; SFCT: subfoveal choroidal thickness; SCP, DCP and CC presented the density measured by OCTA software; SCP',DCP' and CC'showed the density measured by Image J software.

\section{Figures}
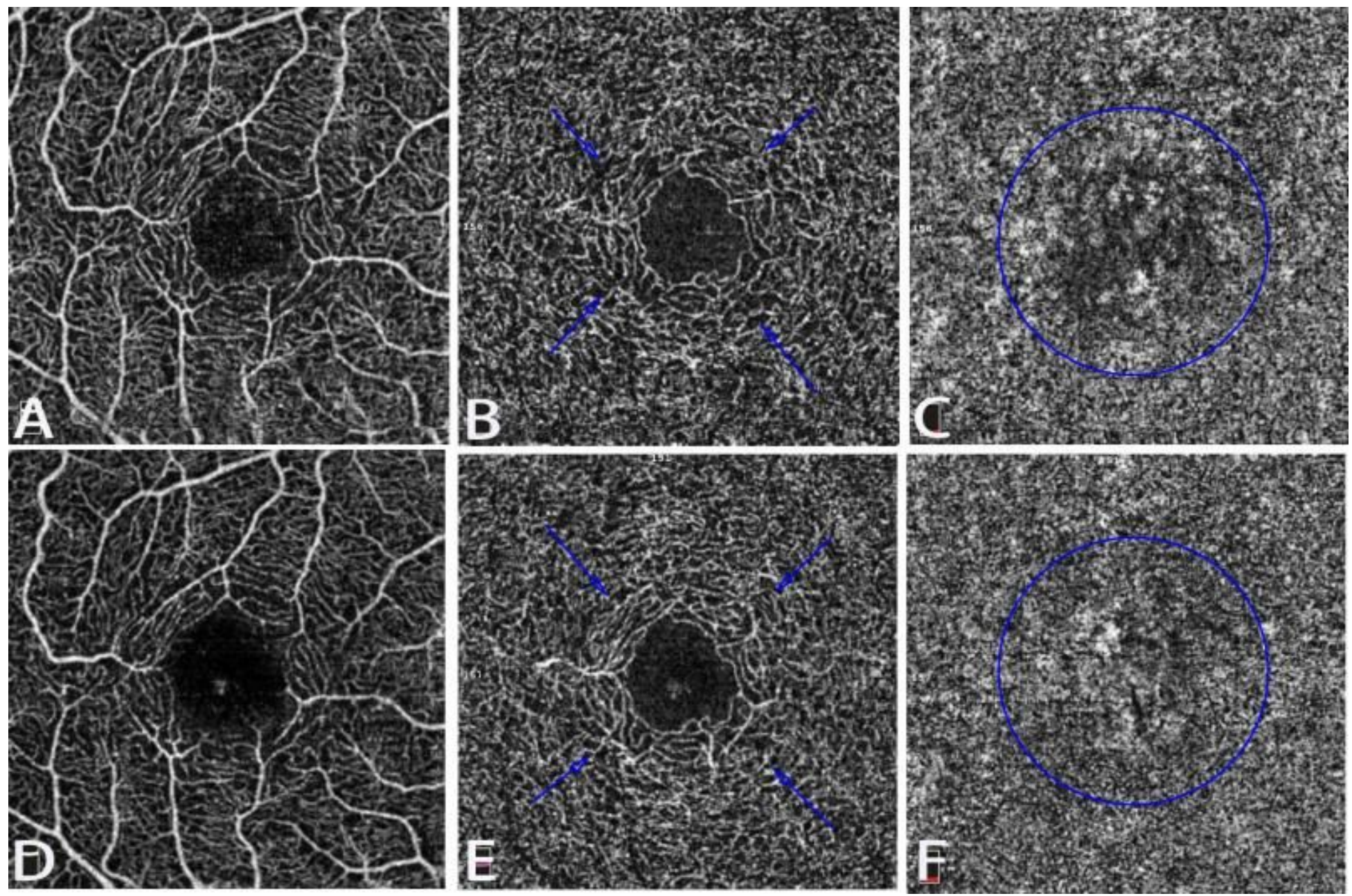

\section{Figure 1}

The manifestation of microvascular in superficial capillary layer (SCP), deep capillary layer (DCP) andchoriocapillary layer (CC) layer at baseline and 3 months. (A): the morphology of SCP at baseline; (B): the morphology of DCP at baseline; (C): the morphology of CC at baseline; (D): the morphology of SCP at 3months; (E): the morphology of DCP at 3 months; (F): the morphology of CC at 3 months; (Blue arrow)the microvascular density of DCP layer at 3 months (E) were increased compared with the one at baseline (B). (Blue circle) the density of CC layer were increased at 3 months compared with the one at baseline. 

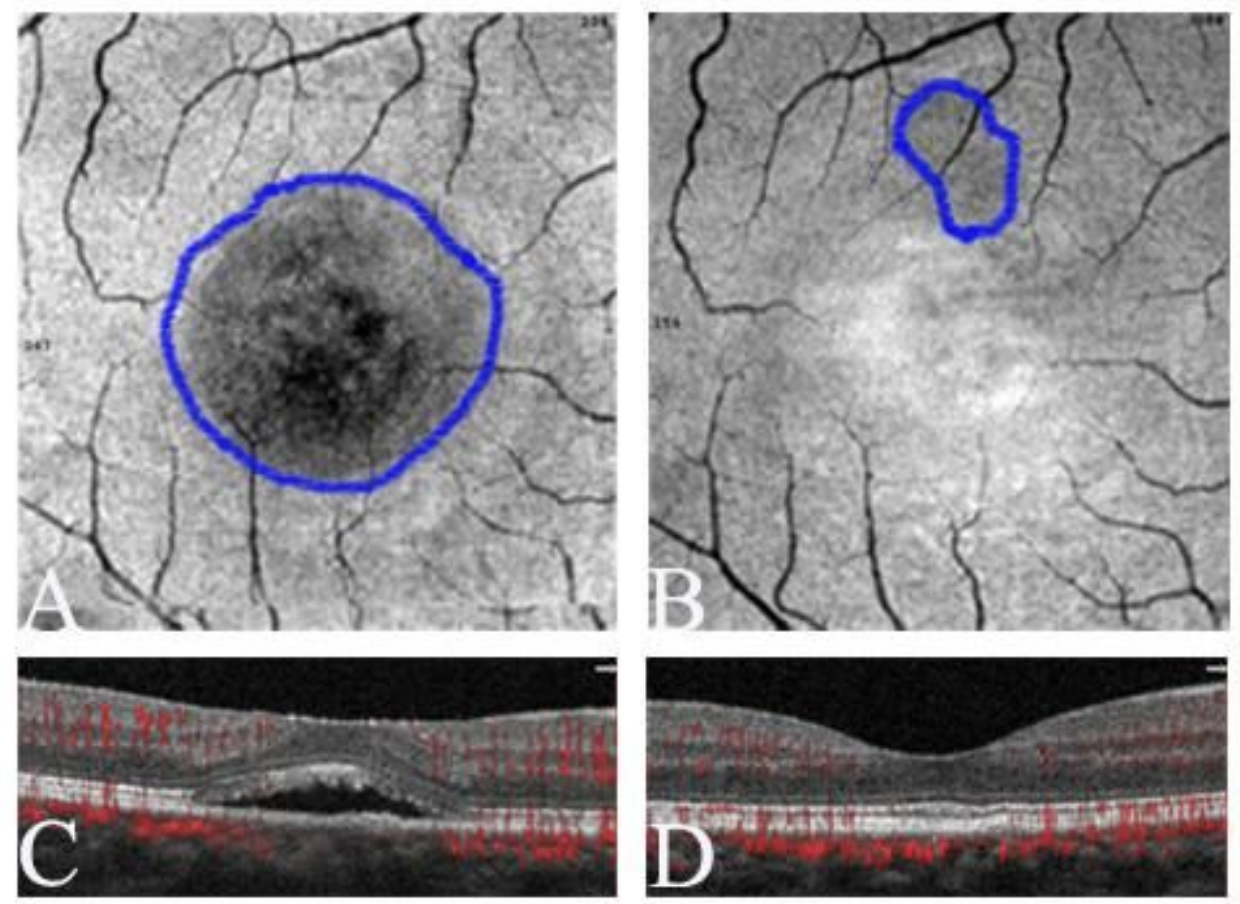

Figure 2

En-face OCT imaging of outer layer in a man at the age of thirty-six by the model of $3 \mathrm{~mm} \times 3 \mathrm{~mm}$ OCTA. A and $B$ showed the en-face of outer layer at baseline and at 3 months. $C$ and $D$ showed the structure of OCT from OCTA at the first of follow-up time and at three months. A stands for the range of photoreceptor disruption in blue circle coresponding the range of fluid accumulation at the picture of $\mathrm{C}$. The range of photoreceptor disruption at picture A was larger than the one of it at picture $\mathrm{C}$. 

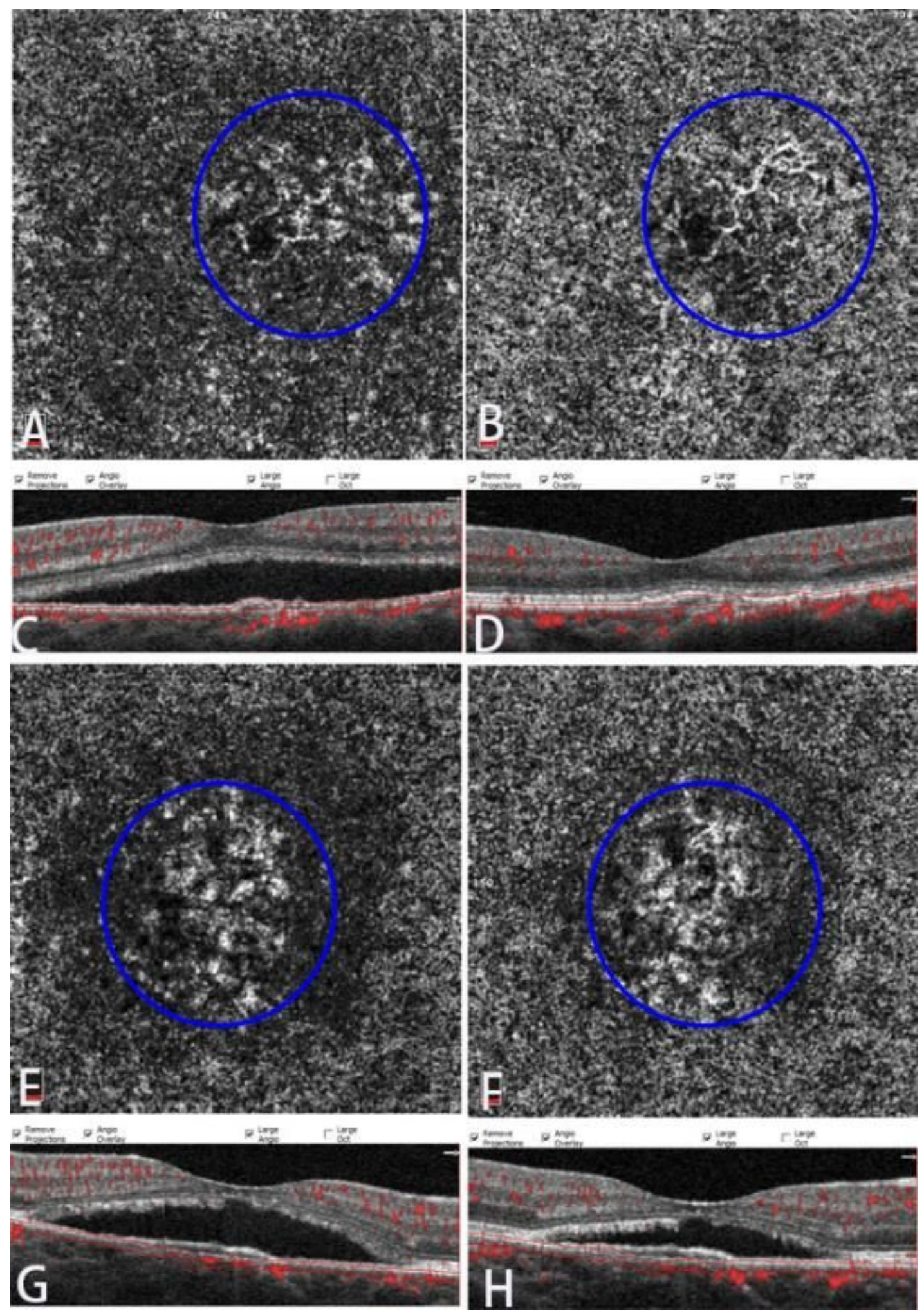

Figure 3

OCT and OCTA imaging of flat PED in 2 females by the model of $3 \mathrm{~mm} \times 3 \mathrm{~mm}$ OCTA. A and E presented locally high signal and peripheral low signal of choriocapillary layer at baseline.B showed remodeling choriodal neovascularization at 3 months. $\mathrm{F}$ manifested much high signal and less low signal at 3 month than at baseline. 

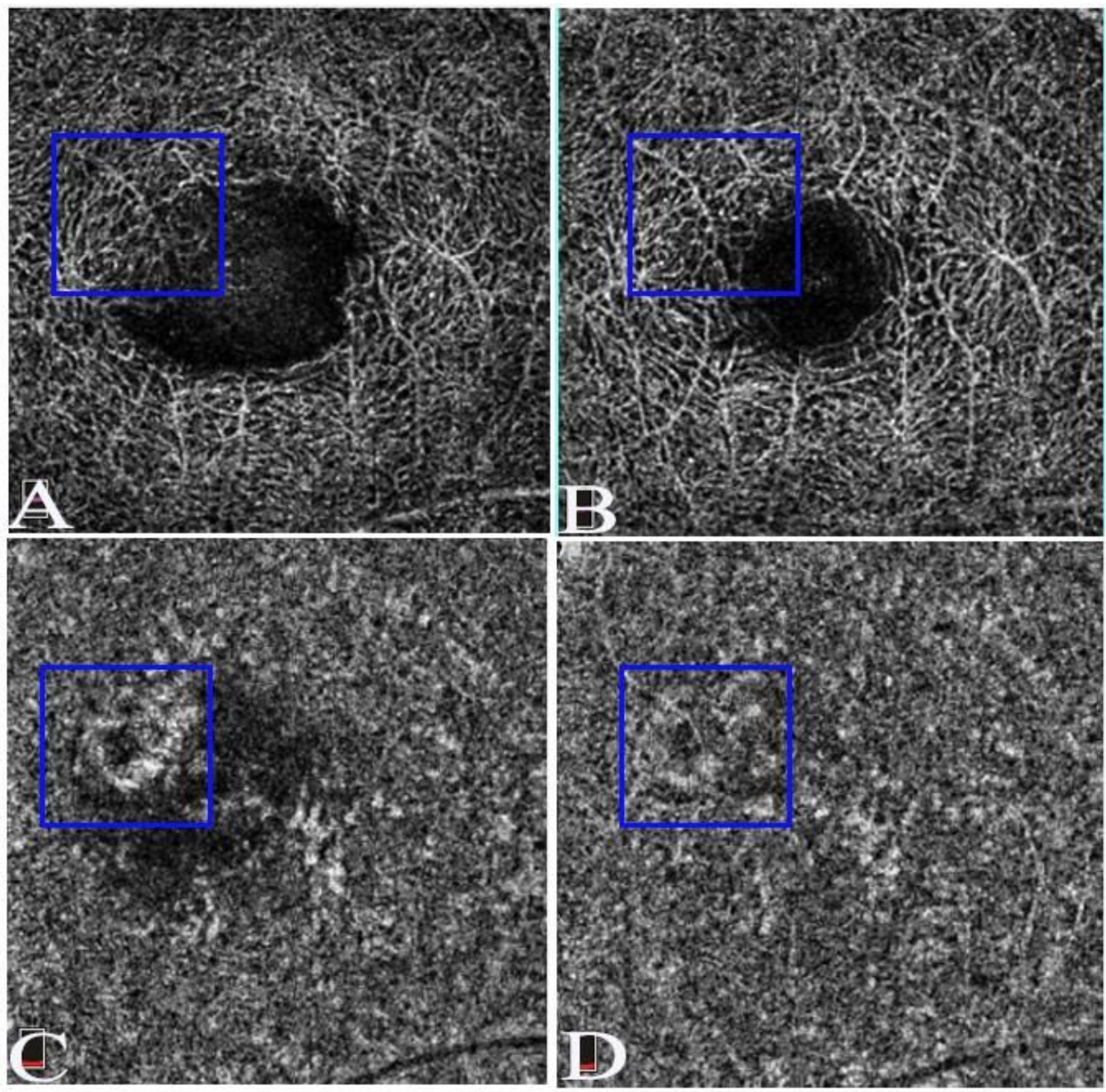

\section{Figure 4}

The vascular morphology of deep layer and choroidal capillary layer in a man at the age of thirty. A and B showed the vascular density of DCP at baseline and at three months, respectively. C and D showed correspondingly angiectasis in choroidal capillary layer at baseline and at three months, respectively. The increased density of deep layer at three months(B) compared with the one of DCP at baseline(A) indicated the recovery of choroidal capillary layer(D) compared with the angiectasis at choroidal capillary layer(C) corespondingly. The region of blue circle stands for the similar position. 